\title{
Experimental Exploration of Multimodal Teaching Mode in College Russian Teaching Under the Mode of Sino-Russian Cooperative Education
}

\author{
Niu Jiping \\ Sino-Russian Institute, Jiangsu Normal University, Xuzhou, China
}

Email address:

xiamenniujiping@126.com

\section{To cite this article:}

Niu Jiping. Experimental Exploration of Multimodal Teaching Mode in College Russian Teaching Under the Mode of Sino-Russian Cooperative Education. Science Innovation. Vol. 6, No. 3, 2018, pp. 123-128. doi: 10.11648/j.si.20180603.12

Received: April 24, 2018; Accepted: June 6, 2018; Published: June 22, 2018

\begin{abstract}
This article applies the multimodal teaching method to college Russian teaching Under the Mode of Sino-Russian cooperative education. Through multimodal classroom teaching experiments, a multimodal teaching model was established. This paper explores the feasibility and effectiveness of this teaching idea in the Russian teaching. The results of experiments show that Under the Mode of Sino-Russian cooperative education the multimodality of Russian teaching helps to fully mobilize the students' enthusiasm for learning, and develop Russian language skills.
\end{abstract}

Keywords: Sino-Russian Cooperative Education, Multimodal Teaching, Russian Teaching,

Experimental Exploration

\section{中俄合作办学模式下多模态大学俄语教学实验探究}

\section{牛继平}

中俄学院, 江苏师范大学, 徐州, 中国

\section{邮箱}

xiamenniujiping@126.com

摘要: 本文将多模态教学方法应用于中俄合作办学模式下大学俄语教学中, 通过开展多模态课堂教学实验, 构建多模 态教学模式, 探析该教学理念在大学俄语教学中的可行性及有效性。教学实验结果表明: 与传统的教学模式相比, 中 俄合作办学模式下多模态大学俄语教学有助于充分调动学生的学习积极性, 培养自主学习能力及俄语应用能力。

关键词: 中俄合作办学模式, 多模态教学, 大学俄语, 实证研究

\section{1. 引言}

随着我国“一带一路”倡议的深入推进，与沿线俄语国 家在经贸、科技等领域的交流合作不断扩大，由此对“专 业+俄语”的复合型人才需求量倍增。加之近年来国内对俄 罗斯教育认同度的不断提升, 中俄合作办学迎来新契机。 在这种办学模式下, 以教师为中心的单一型灌输式教学模
式已不能满足培养高质量俄语复合人才的要求。鉴于此, 探索多模态大学俄语课堂教学, 实现多种模态的相互配合 与协调, 高效提升学生的俄语技能, 对中俄合作办学模式 下的俄语教学既是机遇, 又是挑战。 


\section{2. 多模态教学理念的研究背景}

模态是事物通过一定模式、方式或形式所表现出的属 性或情形 $[1]$ 。不同学科对其划分标准不同, 当代语言学研 究室主任顾曰国指出, 模态指人类通过感官 (如视觉、听 觉等) 跟外部环境之间的互动方式[2]。单个感官称之为单 模态, 而多模态则是通过整合、编排两种以及两种以上的 模态构成一个语篇。纵观国外学者的研究, R.Barthes是多 模态领域最早的研究者之一, 他主要探讨图像在表达意义 上与语言的相互作用。此后, Kress \& van Leeuwen建立了 图像分析的多模态框架, 即研究图像、声音以及颜色在语 篇中的作用 [3]。New London Group将多模态理论应用于教 学, 首次提出了“多元识读能力”, 并指出, 培养学生的多 元读写能力以及解读多模态各种意义的能力是语言教学 的主要任务 [4]。Stein 明确提出了多模态教学法的概念, 即教师和学生都应当利用多模态进行教学和实践, 课堂应 当是包括视觉模态、书面模态、口头模态、表演模态、音 频模态和体态模态在内的多模态符号空间 [5]。此研究可以 对集图像、动作、声音和语言于一身的课堂话语进行较为 全面的分析[6]。2014年6月召开的第七届国际多模态会议 上, 与会专家对多模态的研究方法及发展方向进行了详细 解读。在会议的主旨发言中, 澳大利亚 Kay O'Halloran 博 士介绍了他的团队研发的软件, 如平面媒体、视频及社交 网络等新媒体语篇, 这是多模态研究的新角度 [7]。由此可 见, 英语语言国家对多模态教学的研究已经趋向成熟。

在俄语发展进程中, 最早将图像纳入语言学范畴的是 20 世纪30年代 А.А.Реформатский 编写的《Лингвистика и полиграфия》一书[8]。此后, 该领域的主要研究学者还 有 Е.Е.Анисимова[9], В.М.Березин[10], Л.С.Большиянова[11]等, 其研究视角多为语言符号与非 语言符号在“多符号语篇”中的建构。近年来, 俄罗斯学者 对“外语教学多模态探析”研究兴趣日益浓厚, 在该领域涌 现出诸多学术论文, 例如, 《Мультимодальность в обучении русскому языку как иностранному》 (Коздра Михал), 《Реализация мультимодального подхода к обучению чтению на среднем этапе изучения английского языка》 (Н.А. Фадина)[12]等, 均以注重多模 态读写、交际能力为目标, 旨在提高语言学习效率, 增强 语言学习技能。

近年来, 国内学术界对多模态的研究呈现出极大的热 情。国内较早介绍多模态话语分析的李战子[13]学者指出 了探究多模态话语分析对英语教学的重要意义。顾曰国 [14]、张德禄[15]的研究对推进多模态话语分析起到了全 方位的导向作用。此外，杨信彰[16]、辛志英[17]、张征[18] 等学者为多模态大学英语教学均做出了重要贡献。

因此, 纵观国内外多模态教学的研究成果, 多模态教 学是通过视觉、听觉等多种模态同时刺激学习者的感官, 使其协同运作，以达到强化记忆、提高交际能力的目的[19]。 但是, 总体存在以下几点不足：（1）目前从多模态视角 探析大学外语教学, 主要集中在英语语种, 对俄语、法语、 德语等非通用语种的多模态教学研究较少。(2) 对大学 外语多模态教学的研究多集中在理论方面, 实证研究相对 较少。因此, 本文将从实证角度探析多模态大学俄语教学,
以此验证多模态教学理念在俄语教学中的重要性, 以及该 教学模式对学生的俄语学习兴趣、自主学习能力与俄语语 言应用能力的提升作用。

\section{3. 中俄合作办学模式下多模态大学俄语教学的 必要性}

中俄合作的新型国际化办学模式下, 提高学生俄语应 用能力的目标对俄语教学提出了严峻考验。但现阶段俄语 教学存在着一系列问题: 一是俄语授课教师多为俄语专业 出身, 受其自身的学习痕迹及传统教学方式的影响, 新模 式下一时很难改变传统教学方法; 二是学生对合作办学模 式的理解不够透彻, 加之学生多属于俄语零起点, 导致俄 语学习的自主性较差; 三是俄语教材的编排缺乏针对性、 趣味性, 仅有录音, 文本等单一模式。以上诸多因素, 阻 碍了学生俄语学习效果的有效提升。因此, 探索多模态大 学俄语教学, 不仅是中俄合作办学俄语教学改革的必需, 也是培养复合人才多元识读能力的要求。

\section{1. 中俄合作办学模式下俄语教学改革的必需}

目前, 大学俄语教学大致分为三种类型：专业俄语教 学、公共俄语教学、复合型俄语教学。中俄合作办学的俄 语教学区别于大学专业俄语教学及公共俄语教学, 以江苏 师范大学中俄学院金融工程专业为例, 其培养目标在于, 培养金融专业知识扎实、俄语水平高、操作能力强, 且熟 知中俄两国金融知识的国际化、复合型高级专门人才。因 此, 学生的俄语应用能力和金融词汇的熟练掌握应当成为 金融工程专业俄语教学的重中之重。在该特色化的培养目 标下, 课堂上仅使用单一的听觉或视觉模态教学, 很难在 “3+1”的学制下培养并提升学生的俄语应用能力。而多模 态理论则为金融专业俄语教学改革提供新启示: 教师既要 充分利用多媒体教学条件, 最大限度调动学生以听觉、视 觉等模态为主的多模态学习, 同时加入动画、图像等辅助 模态, 开展积极、高效、有趣的语言学习。中俄合作办学 模式下的多模态教学为俄语课堂注入新生, 提高课堂效率, 优化教学效果。

\section{2. 培养复合人才多元识读能力的要求}

近年来，随着中俄战略合作的不断加深，我国与俄语 国家的经贸往来日益频繁。传统的印刷品随着多媒体时代 的到来, 已经无法满足社会发展需要; 与之相反, 通过俄 文网站获取最新资料、了解国家间的最新经贸信息已经普 及。因此, 中俄合作办学模式下培养复合型人才的多元识 读能力势在必行——学生不仅要具备文化识读能力, 也要 具备多模态识读能力。为实现这一目标, 将多模态俄语教 学运用到课堂中尤为关键, 教师通过运用图片、网络、角 色扮演等多种方式, 培养起学生阅读多媒体与模态所提供 的信息的能力。学生通过理解多模态环境下的各种经历, 建构起自身对现实世界的认识, 继而能够达到自主学习的 目的, 以迎接经济全球化的挑战。 


\section{4. 多模态大学俄语教学的实证研究}

本文以多模态话语理论为基础, 结合当前大学俄语 面临的困境及出现的问题, 着重从教师和学生两个角度 对多模态大学俄语教学模式从三个方面进行建构: 第一,
教师多模态话语的运用在多模态大学俄语教学实践中 的重要作用; 第二, 学生在多模态大学俄语教学实践中 学习兴趣的培养以及语言应用能力的提升; 第三, 大学 俄语多模态教学的评估标准。具体图示如下:

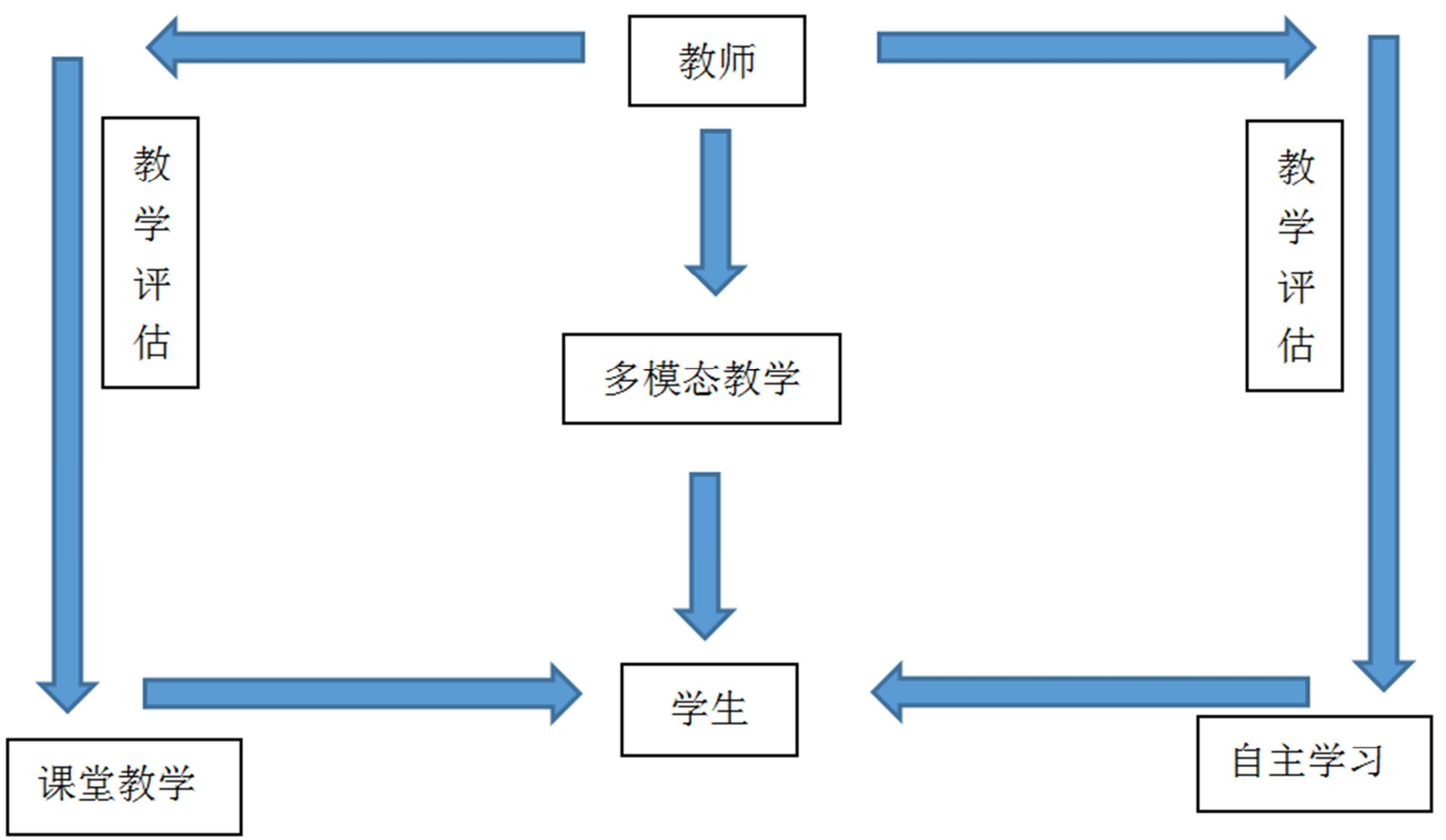

图1 模态俄语教学模式。

\section{1. 教学实验}

\subsection{1. 研究目的}

本研究目的在于: 验证多模态教学在中俄合作办学模 式下大学俄语教学中的有效性, 提高俄语课堂教学效果, 培养学生的俄语学习兴趣及语言运用能力。

\subsection{2. 研究方法}

本研究采用定量研究和定性研究相结合的方法。首先 对实验班进行多模态教学前测, 经过两个月的多模态俄语 教学后, 再对其进行后测, 将前测和后测成绩进行对比, 分析大学多模态俄语教学模式对学生俄语学习能力、多元 识读能力的影响。

\subsection{3. 研究对象}

本研究选取江苏师范大学中俄学院金融工程专业某班 级作为实验班, 该班学生人数为 31 人。对同一个实验班进行 了为期两个月的多模态教学实验。为了确保实验的严谨性, 此次教学实验确保学生在不知道自己参与的情况下进行操 作。实验前采用传统俄语教学法, 实验中采用多模态教学。

\subsection{4. 研究工具}

课本: 本实验选用《走遍俄罗斯 2 》作为使用的教材, 该书有七节内容。通过仔细分析与思考, 选取本书的一、 二节作为研究材料。
PPT演示稿: 根据课程内容的多样性, 每节课将设计 和制作不同内容的PPT演示稿。PPT演示稿涉及到多模态 符号, 如: 图片、文字、视频和声音等。且实验过程中注 重动画及视频的插入。

视频和音频：实验期间增加动画视频环节，此处以 《走遍俄罗斯 2 》第二节语法知识点——运动动词为例, 并将俄罗斯动画片《Малышарики》第35集Поехали!作 为该课程的课堂导入部分, 为学生创建视觉模态, 培养 学生对运动动词学习兴趣及理解能力。同时, 课堂上教 师使用与《走遍俄罗斯》教材配套的多媒体光盘为学生 创建听觉模态。此外, 这些资源可帮助学生课后进行自 主学习。

网络自主学习平台: 为学生推荐难度适宜的网络学习 软件, 图片、文字、声音等方式便于学生对知识的理解和 消化, 同时弥补教材过于单一、知识点零碎的缺陷。

试卷: 这项研究是同一班的不同时间进行的。本文用 半学期时间对实验班开展研究, 前测及后测题型及难度均 符合教学大纲。

\subsection{5. 研究分析过程}

借助社会科学统计软件SPSS24.0对所收集到的前后 测成绩进行数据统计, 使用成对样本 T检验的统计方法, 对比实验班前测及后测俄语水平测试差异。以下表 $1 \sim 3$ 是 俄语水平能力测试成绩成对样本统计的具体数据。 
表1 综合俄语水平能力测试成绩配对样本统计量。

\begin{tabular}{llllll}
\hline 均值 & & $\mathbf{N}$ & 标准差 & 标准误差平均值 \\
\hline \multirow{2}{*}{ 配对 1} & 前测成绩 & 78.55 & 31 & 15.782 & 2.834 \\
& 后测成绩 & 81.52 & 31 & 13.015 & 2.338 \\
\hline
\end{tabular}

表2 综合俄语水平能力测试成绩配对样本相关系数。

\begin{tabular}{lllll}
\hline $\mathbf{N}$ & & & 相关性 & 显著性 \\
\hline 配对 1 & 前测成绩 \& 后测成绩 & 31 & 0.975 & 0 \\
\hline
\end{tabular}

表3 综合俄语水平能力测试成绩配对样本检验。

\begin{tabular}{|c|c|c|c|c|c|c|c|c|}
\hline & \multicolumn{5}{|c|}{ 成对差值 } & \multirow{3}{*}{$\mathbf{t}$} & \multirow{3}{*}{ 自由度 } & \multirow{3}{*}{ 显著性 (双尾) } \\
\hline & \multirow{2}{*}{ 平均值 } & \multirow{2}{*}{ 标准差 } & \multirow{2}{*}{ 标准误差平均值 } & \multicolumn{2}{|c|}{ 差值 $95 \%$ 置信区间 } & & & \\
\hline & & & & 下限 & 上限 & & & \\
\hline 配对1 & -2.968 & 4.223 & 0.758 & -4.517 & -1.419 & -3.913 & 30 & 0.000 \\
\hline
\end{tabular}

从配对样本统计量表 1 来看, 前测成绩的均值是 78.55 , 后侧成绩的均值是 81.52 , 后侧成绩比前测成绩好。

原假设：配对组间没有相关性；备择假设：配对组间 有相关性; 显著性值在表格中显示为 0.000 , 其意为该数 值远小于千分之一, 在给定显著水平 0.05 情况下, 因此可 以拒绝原假设, 即配对组间有相关性。

从配对样本相关系数表 2 来看, 相关系数为 0.975 , 说 明配对组之间属于强相关, 因此可以用配对样本检验。配 对样本 $\mathrm{T}$ 检验的结果, 分析最后三列: $\mathrm{T}=-3.913$, $\mathrm{DF}=30, \mathrm{P}<0.001$ 。在给定显著水平为 0.05 的情形下, 可以认 为配对组间的均值差异有统计学意义。

从成对差值表 3 来看, 即表 3 的 4,5,6列, 如果 $95 \%$ 置信 区间不包含 0 , 则 $P<0.05$ 同样可以得出结论 2 组差异具有统 计学意义。

此外, 本文还对学生的总体成绩进行了以下统计(见 图1、图2)。通过比较可得知, 后测成绩中不及格的比例 $6 \%$, 较前测成绩 $19 \%$ 明显降低。

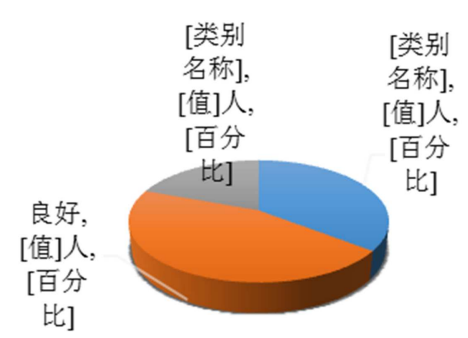

曰优秀曰及格、不及格

图2 前测总成绩。

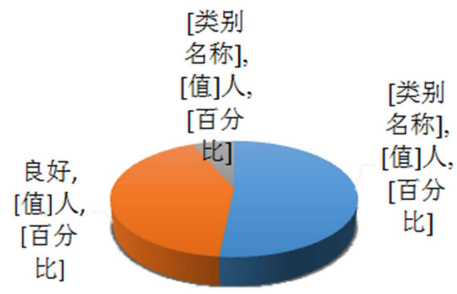

曰优秀匹及格匹不及格

图3 后测总成绩。

\subsection{6. 研究结果}

上述统计结果分析显示: 利用配对样本 $\mathrm{T}$ 检验来判断, 综合俄语水平测试前后测成绩的均值差异有统计学意义, 认为后测成绩优于前测成绩。因此, 可以认为在接受了大 学俄语多模态教学模式的实验后, 实验班在第二阶段的俄 语水平得到提高, 且两极分化情况有所减弱。

\section{2. 访谈分析}

通过教学实验可以分析出多模态俄语教学促进了学 生俄语学习能力的提升, 为了更全面地检验该结果, 在教 学实践和实验结束后, 本文对实验班的一些学生进行访谈, 访谈的目的是调查学生对多模态教学和传统教学模式的 态度。访谈内容如下:

(1) 你觉得现在的综合俄语课与上学期相比, 在教师授 课及学生学习方面有什么不同?

(2) 你喜欢老师课堂上运用的PPT课件、图片、音乐、视 频、动画等授课形式吗? 是否对你的学习有帮助?

（3）相比于传统的俄语课, 逐字逐句讲解语法、词汇、 课文, 你是否适应新型的多模态教学法?

访谈结束后对访谈内容进行归纳分析: 第一, 多数同 学表示, 现在的课堂丰富多彩比以前有趣, 且气氛更加活 跃, 听说等交流互动增多; 第二, 受访得所有学生都非常 喜欢教师课堂上使用的PPT课件、视频、音乐, 尤其是动 画片备受部分学生的喜爱, 这些方法能够激发他们的学习 兴趣, 激发学习动力, 一些学生还指出课外视频材料增进 了自己对俄罗斯的了解; 第三, 与传统的俄语课上逐字逐 句讲解语法、词汇、课文相比, 学生们青睐新型的多模态 教学法。

通过上述访谈结果的分析得出结论, 视觉和听觉等多 种模态结合起来进行信息交流与理解, 要比单一听觉模态 有效得多。多模态俄语教学模式能够弥补传统单一教学模 式的不足, 多模态材料的输入可以激发学生的学习兴趣, 提供视频、图片等直观、逼真的教学资源, 能够增强学生 对知识的理解, 同时多种模态相互融合有助于培养学生多 元识读能力[12]。 


\section{5. 多模态大学俄语教学的启示}

\section{1. 教师要开展俄语课堂多模态教学实践活动}

通过实验发现, 多媒体技术在大学俄语多模态教学中 发挥着重要作用。多模态课堂的特色在于教学多模态化策 略的合理使用。它克服了传统俄语课堂“以逐字逐句讲解 词汇句型语法为特征”的单模态模式, 因而改变了灌输式 俄语教学单调、沉闷的教学气氛, 增加了教师与学生之间 的交流互动。

\section{2. 学生要参与主动到多模态教学}

利用多媒体 PPT 辅助教学, 将图像、视频、音乐、 动画等多模态符号植入课堂, 多种模态相互协作, 吸引学 生的注意力, 充分刺激他们的视觉和听觉, 激发学生学习 兴趣, 培养学习动力, 有助于培养学生俄语应用能力。学 生在有趣的俄语学习环境中, 高度融入课堂, 培养学生多 元识读能力。

\section{3. 俄语课堂多模态选择坚持最优与适配原则}

通过学生的建议, 在进行访谈的过程中也发现了部分 问题, 比如, 课堂不能为单纯追求多媒体和多模态的教学 形式而滥用无效的媒体技术。毕竟课堂时间是宝贵的，过 度注重课堂互动环节以及进行视听资料的播放有时会拖 慢课程进度。因此, 在进行多模态选择时要兼顾最优与适 配原则, 一方面, 应该将模态以不同的方式组合, 以达到 不同模态互相协调配合、相互转化, 实现多模态学习的目 的。另一方面, 多模态组合时要把握好模态选择的尺度, 坚持适配原则, 如果模态以不适当的方式组合在一起, 有 可能会降低课堂教学的整体效应[20]。

\section{4. 强化多模态课外自主学习}

多模态自主学习是指学生依据教学要求和教学任务, 根据自己的学习水平和学习方法, 在仿真性或真实性语言 环境中通过视觉、听觉和触觉多种模态认知、处理、接收 和运用语篇信息和非语篇信息[21]。仅仅借助课堂运用多 模态是远远不够的, 因此, 学生可以通过网络平台强化多 模态自主学习, 才能使多模态教学具有延续性。网络学习 平台主要通过俄语网络平台、网络课程、俄语视听说教学 资源库等为学生构建多模态仿真实践环境。最大化地实现 学习者和多媒体语料之间的实现学习者和多媒体语料之 间的多模态互动。

\section{6. 结论}

本研究结果表明, 中俄合作办学模式下大学俄语多模 态教学模式的科学性, 可行性及有效性在教学实践中得到 了验证。首先, 借助多媒体与网络技术, 多模态俄语教学 为俄语课堂提供高质量的语言交际语境, 它通过调动学生 的多种感官来激发学生的学习动机和兴趣, 有助于培养学 生俄语应用能力; 切实帮助学生提高现代交际所必需的俄 语多模态听说读写能力, 以应对经济全球化、语言文化多
元化和交际多模态化所带来的挑战; 同时，对于多媒体时 代中俄合作办学模式下大学俄语教学的发展和走向也有 一定的借鉴作用。但是, 在实验过程中本研究采用的样本 数量过少以及周期相对较短, 因此还存在着一定的局限性。 本研究的缺陷和不足有待于后期研究和其他学者相关研 究的补充和完善, 以促中俄合作办学模式下大学俄语教学 质量的高效提升。

\section{参考文献}

[1] 郭万群.大学英语多模态课堂教学研究 $[M]$.上海: 上海交通 大学出版社,2015。

[2] 顾曰国.多媒体、多模态学习剖析 [J]. 外语电化教学,2007, (4) : $3-12$ 。

[3] Kress G, Van Leeuwen T. Reading Images: The Grammar of Visual Design.[M]. London: Routledge, 1996.

[4] The New London Group. A Pedagogy of Multiliteracies: Designing Social Futures.[M].London: Routledge, 1996.

[5] Stein. Rethinking resources: Multimodal pedagogies in the ESL classroom.[J].TESOL QUARTERLY, 2000, 34: 333.

[6] 马冰鹤.多模态在大学英语教学中的应用 [J].吉林医药学院 学报,2018,39(02):158-159。

[7] 秦琼芳,徐航.多模态虚拟课堂——法语文化类课程教学新 模式探究[J].牡丹江教育学院学报,2017(11):62-63。

[8] Реформатский А.А. Лингвистика и полиграфия // Письменность и революция. М. ; Л.,1933Коздра Михал. Мультимодальность в обучении русскому языку как иностранному.[М].Польша. 2017

[9] Анисимова Е. Е. Лингвистика текста и межкультурная коммуникация(на материале креолизованных текстов): Учеб. пособие для студ. фак. иностр. яз. вузов.[М].:Издательский центр Академия,2003,- С.8.15.

[10] Березин В.М. Массовая коммуникация: сущность, каналы, действия. [M]., 2003.

[11] Большиянова Л. М. Внешняя организация газетного текста полокодового характера // Типы коммуникации и содержательный аспект языка. [М].,1990. С. 180.

[12] Н.А. Фадина. Реализация мультимодального подхода к обучению чтению на среднем этапе изучения английского языка.[М].Саратов. 2017

[13] 李战子.多模式话语的社会符号学分析 [J].话语分析外语研 究.2003,(8):1-8。

[14] 顾曰国. 多媒体、多模态学习剖析[J]. 外语电化教学,2007, (4) : 3-12。

[15] 张德禄. 多模态综合理论框架探索 [J]. 中国外语 2009,(1):24-30。 
[16] 杨信彰.多模态语篇分析与系统功能语言学 [J]. 外语教学. 2009, (7)：11-14。

[17] 辛志英.话语分析的新发展一一多模态话语分析[J].社会科 学辑刊.2008,(5):208-211。

[18] 张征.多模态PPT演示教学与学生学习绩效的相关性研究 $[\mathrm{J}]$. 中国外语2010,(3):54。
[19] 黎健. 大学英语多模态教学探析 [J]. 重庆与世界 (学术 版),2012,29(05):59-61+69。

[20] 易舒.多模态条件下的德语听力教学实践 [J]. 西部素质教 育,2016,2(07):142-143。

[21] 宋英.大学生英语多模态读写能力的培养路径研究 [J].西南 交通大学学报(社会科学版),2018,19(01):74-81。 